\title{
A SPECTRUM OF CLINICAL SIGNS IN ANTERIOR UVEITIS
}

\author{
H. S. DUA, A. D. DICK, N. J. WATSON and J. V. FORRESTER \\ Aberdeen
}

\begin{abstract}
SUMMARY
The range of clinical changes that occur in posterior uveitis have been well documented. We describe two clinical features of anterior uveitis which we have termed 'iris pigment epithelitis' and 'iris vasculitis'. Other clinical forms of iritis also exist and together with our described findings parallel the spectrum of clinical presentations in posterior uveitis.
\end{abstract}

Both anterior and posterior uveitis can be classified as either 'exogenous', when a direct infectious agent can be identified (e.g. herpes virus), or 'endogenous', which represents the majority of uveitides. Evidence suggests that endogenous anterior and posterior uveitis are pathologically distinct. Anterior uveitis in over $50 \%$ of cases is linked to MHC class I autoantigens, ${ }^{1}$ and although probably autoimmune in nature, is most closely linked to a possible underlying infectious aetiology. ${ }^{2}$ On the other hand, the posterior uveitis group is, in the main, linked with MHC class II autoantigens, and autoimmunity. However, birdshot choroidopathy has a high association with the MHC class I autoantigen HLA-A29. ${ }^{3}$ The significance of these underlying immunogenetic differences is not clear, as both MHC class antigens may mediate an autoimmune state, perhaps as a consequence of molecular mimicry from previous antigenic stimulation by an exogenous infection.,

In both the animal model and human disease the clinical signs are a manifestation of the underlying pathological changes. ${ }^{6}$ Posterior uveitis may affect specific regions of the posterior segment (e.g. pars plana, choroid, retina) and more specifically the retinal pigment epithelium, or may present as a panuveitis. Subgroups of posterior uveitis present with common clinico-pathological changes of vitritis, chorioretinal infiltrates, retinal vasculitis and macular oedema. The clinical nomenclature of these posterior uveitides merely denotes the principal clinico-pathological change that is present in a spectrum of posterior segment inflammatory disorders.

Clinical documentation of iritis is not so clearly defined. Types of iritis are largely grouped by the extent

Correspondence to: Dr. Andrew D. Dick, Department of Ophthalmology, Medical School, Foresterhill, Aberdeen AB9 2ZD, UK. and severity of the anterior segment inflammation, such as the form of keratic precipitate that occurs (granulomatous and non-granulomatous), with little other documentation of the clinical changes that may occur.

We describe two subgroups of anterior uveitis, which we defined by the predominant presenting clinical feature. We have termed these groups, 'iris pigment epithelitis' and 'iris vasculitis', and compare these changes with the spectrum of features seen in posterior uveitides.

\section{CASE REPORTS}

We describe two groups of patients who presented with anterior uveitis to the eye clinic at the Aberdeen Royal Infirmary over a 4-year period.

\section{Group A: Iris Pigment Epithelitis}

Group A consisted of 15 patients who presented with a cluster of features associated with anterior uveitis. The iritis was low grade, uniocular, with fine keratic precipitates and occasional posterior synechiae. The major distinguishing feature, however, was focal transillumination defects at the level of the iris pigment epithelium indicating iris pigment epithelial cell loss. Twelve of the patients had a history of recurrent uveitis, 4 had a history of herpes simplex labialis and 4 had a history of varicella zoster infection, but there was no active keratitis or herpetic mucocutaneous lesions on presentation with anterior uveitis. Iris pigment epithelial loss is seen in zoster keratouveitis, frequently associated with stromal atrophy.?

Iris pigment epithelial loss occurred either in the midiris as small defects or as confluent defects that extended to the sphincter and periphery of the iris (Fig. 1a-f). There appeared to be no stromal atrophy in any of these cases. The iris pigment epithelial cell loss was thought to be distinct from other causes of transilluminatory defects (e.g. pigment dispersion) in both distribution and extent. Iris transilluminatory defects do occur in iritis, and must be excluded. They are commonly secondary to posterior synechiae and are thus located around the iris sphincter (Fig. 2 ). We have detailed two case reports to document the iris pigment cell loss in evolution, during a presentation of acute iritis.

Case 1. Mr. RH is a 54-year-old man who presented 

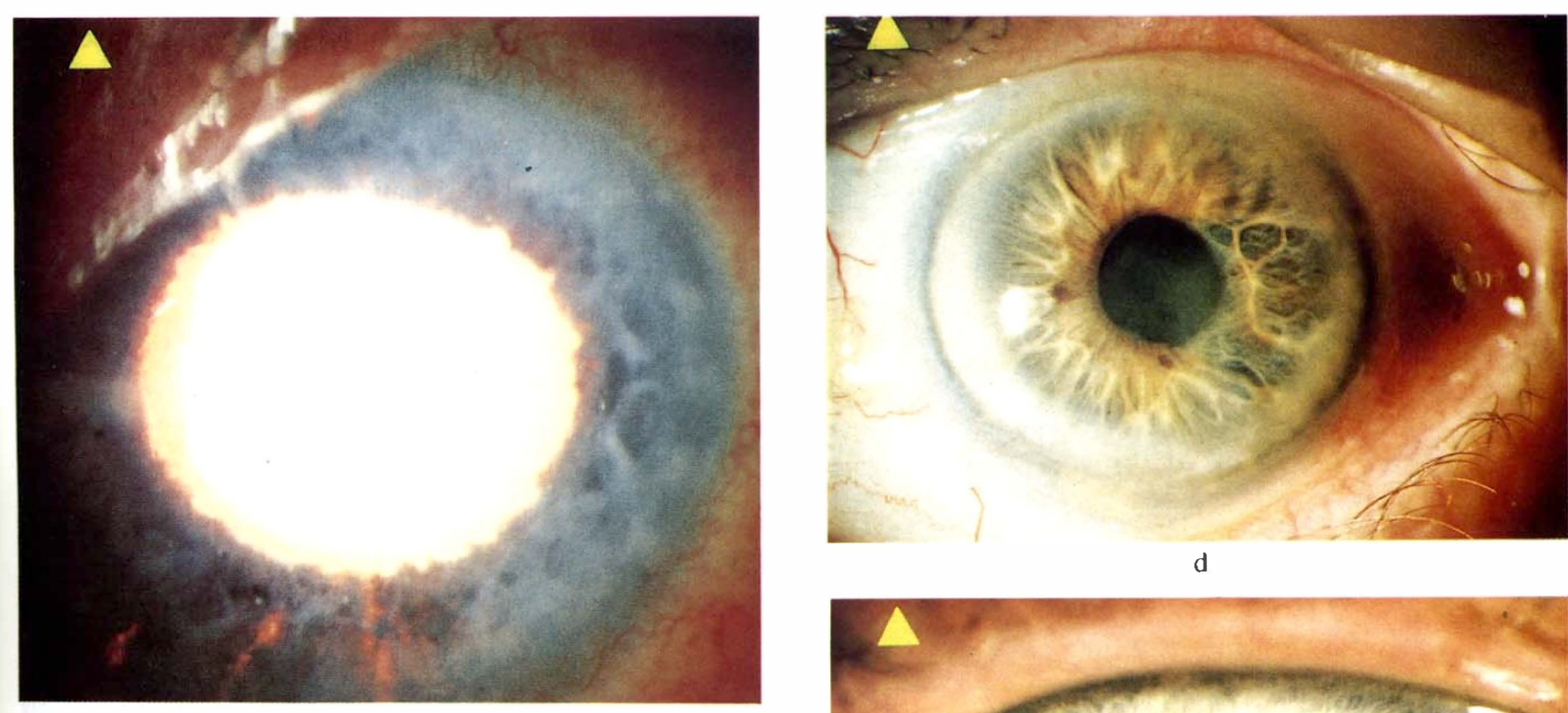

d

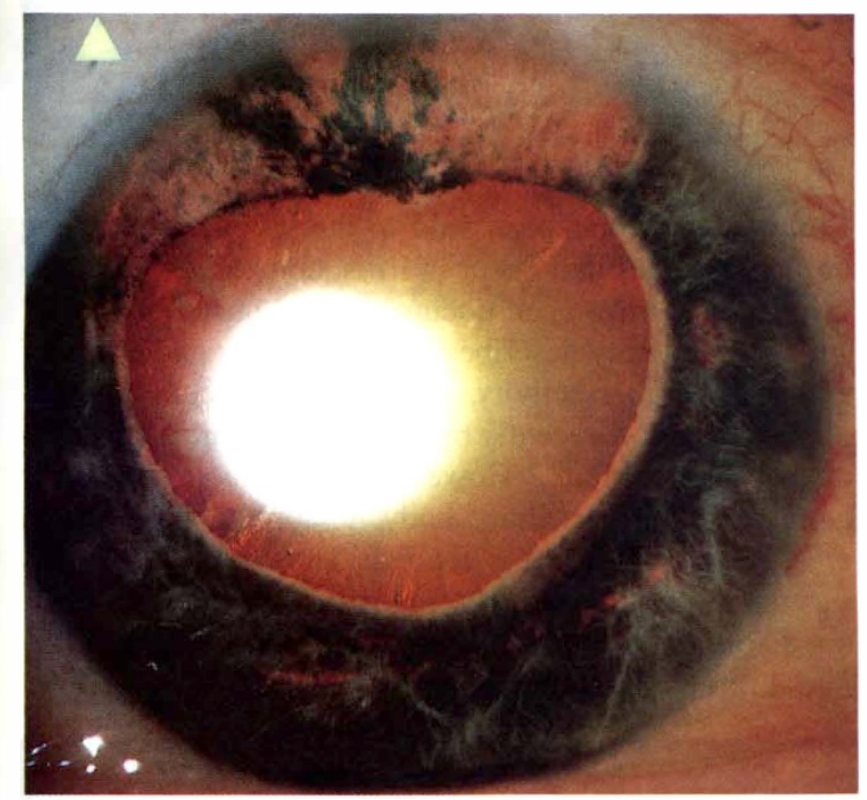

b
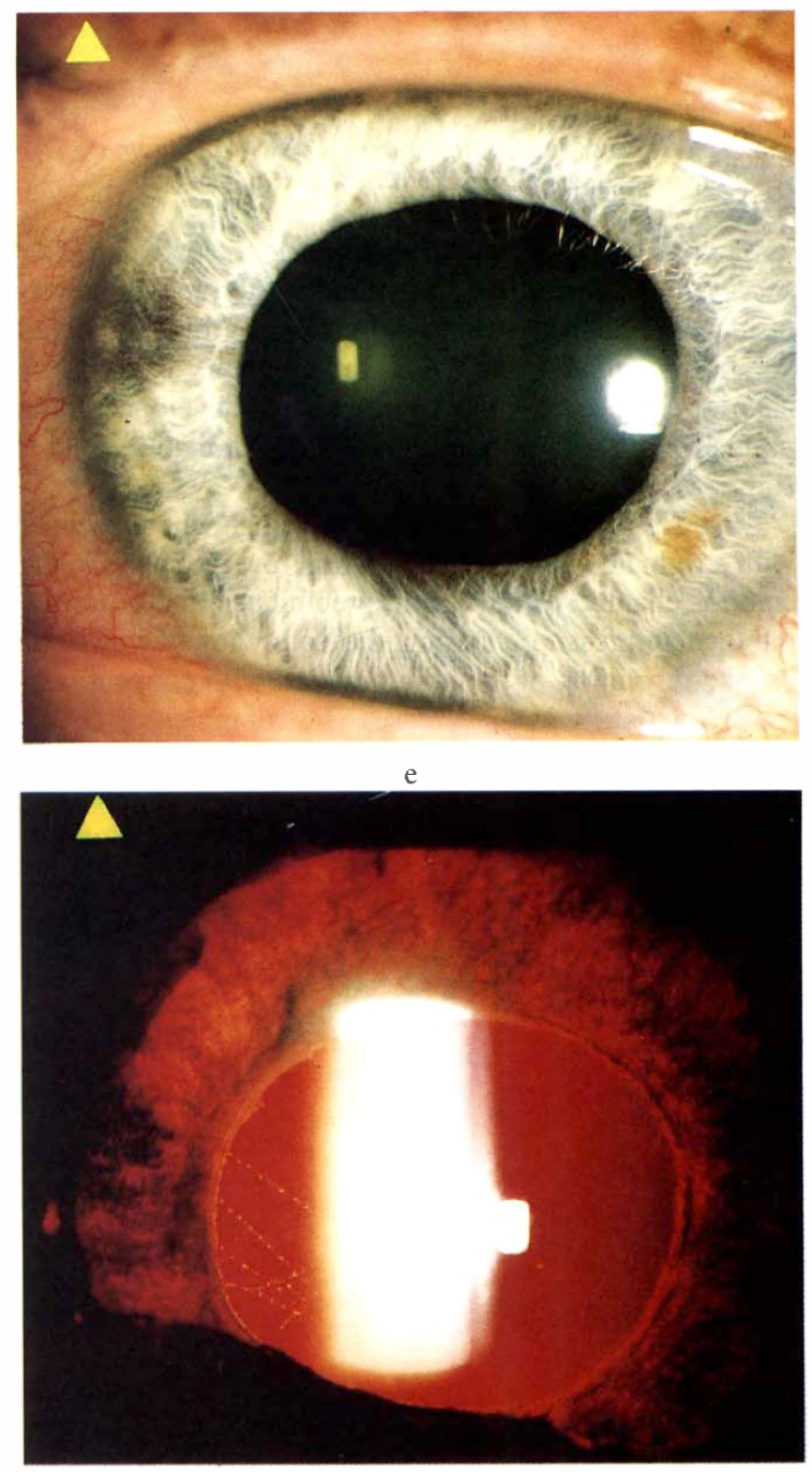

$\mathrm{c}$

$\mathrm{f}$

Fig. 1. Clinical appearance of iris pigment epithelial loss. (a) Scattered iris pigment epithelial loss in the mid-iridal zone. (b) Isolated and confluent iris pigment epithelial loss. $(c)$ and $(d)$ Sectorial iris pigment epithelial loss $(c)$ in the absence of stromal atrophy $(d)$. (e) Anterior segment photograph showing the absence of stromal atrophy. $(f)$ Transillumination of the iris in $(e)$ demonstrating the gross iris pigment epithelial loss. 


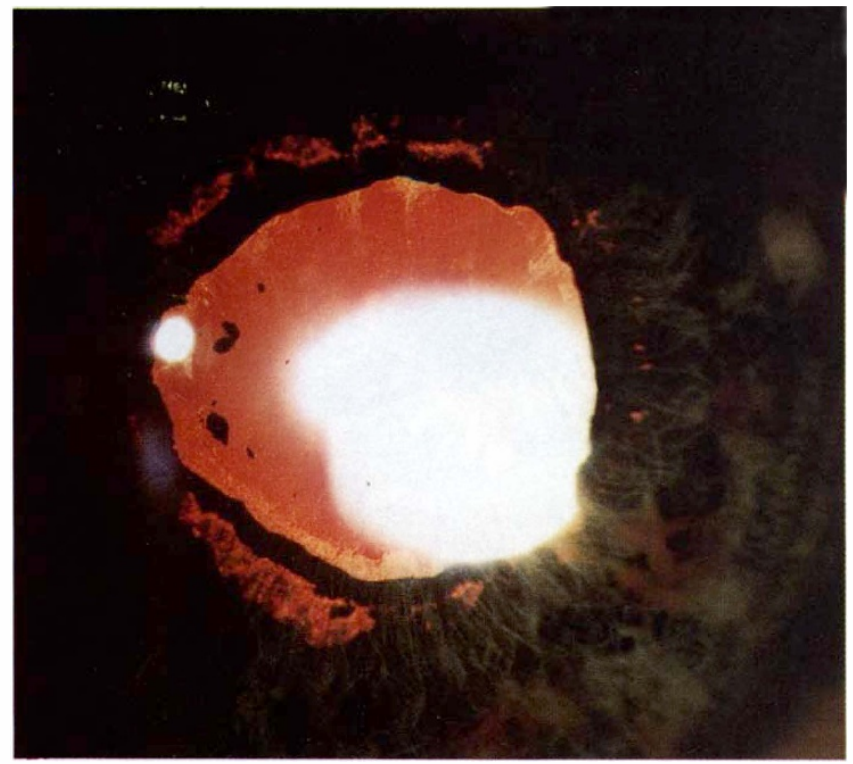

Fig. 2. Causes of iris pigment epithelial loss: pigment epithelial loss secondary to posterior synechiae.

with a right idiopathic posterior scleritis. One week after successful treatment with intravenous steroids, he developed a right anterior uveitis, with multiple fine pigmented keratic precipitates and $2+$ of inflammatory cells in the anterior chamber. Within 1 week he had developed a fine pigment dispersion and focal iris transillumination defects (Fig. 3a, b).

Case 2. Mrs. SS is a 42-year-old woman who presented with a right acute iritis. Examination revealed cells in the anterior chamber, fine keratic precipitates and a normal intraocular pressure. During the following few weeks, despite resolution of the inflammation with conventional treatment, there was an increase in loss of iris pigment epithelium demonstrated by confluent transilluminatory defects at the level of the iris pigment epithelium (Fig. 4a). Iris angiography demonstrated vascular leakage of flu-

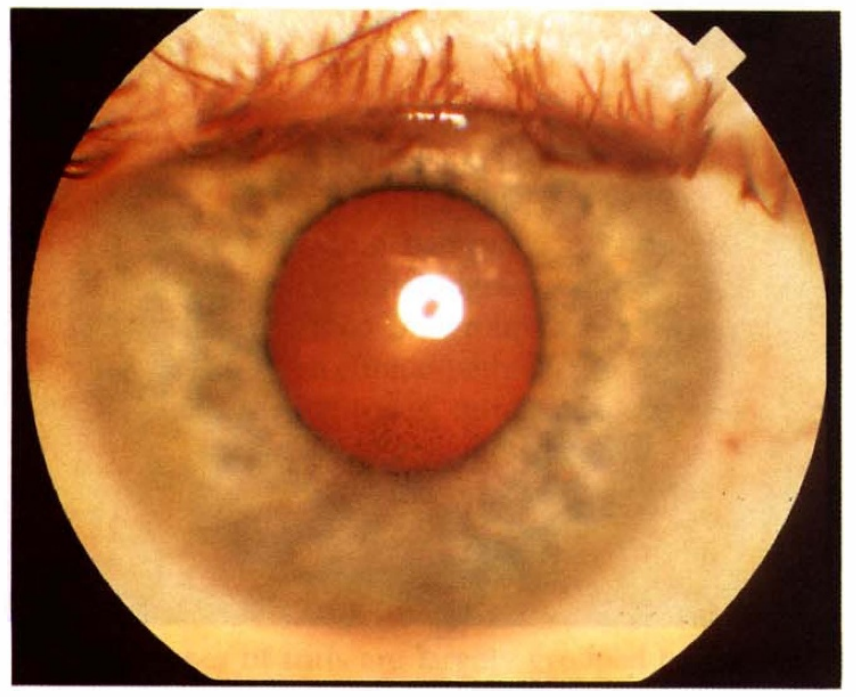

a orescein, although no vascular engorgement or vasculitis was clinically apparent (Fig. 4b).

\section{Group B: Iris Vasculitis}

Group B consisted of 5 patients with a past history of recurrent anterior uveitis who presented with a further attack of acute anterior uveitis. The striking clinical sign which distinguished this group was coiled, swollen, tortuous iris vessels; 4 patients had hyphaema, as well as other classical signs of iritis. In none of these patients was the intraocular pressure raised during the acute phase, nor was there any associated systemic pathology. They all responded well to routine medical therapy.

Fig. 5a and $b$ documents the clinical appearance of these eyes, with coiled vessels and spontaneous haemorrhages from several iris vessels. Iris angiography in the acute, phase revealed the extent of vessel engorgement and the late leakage of fluorescein from the vessel wall (Fig. $6 a, b)$. Angiography performed on one patient 6 months after his acute episode of 'iris vasculitis' demonstrated continued engorgement and tortuosity of these vessels and late leakage of dye (Fig. 7). Clinically there were no signs of intraocular inflammation.

\section{DISCUSSION}

Little attention has been paid to the specific underlying clinical changes that occur in iritis, compared with those changes that have been documented in posterior uveitis. Iritis is frequently described by the severity and extent of anterior chamber activity, particularly the cellular component, and more recently by quantitating flare using automated 'flare meters' ${ }^{8}$ However, there are several other components to iris/ciliary body inflammation and these are highlighted in these reports. The sequelae of iritis include posterior synechiae, secondary glaucoma and cataract formation, and these may vary depending on the initial presentation in the acute phase.

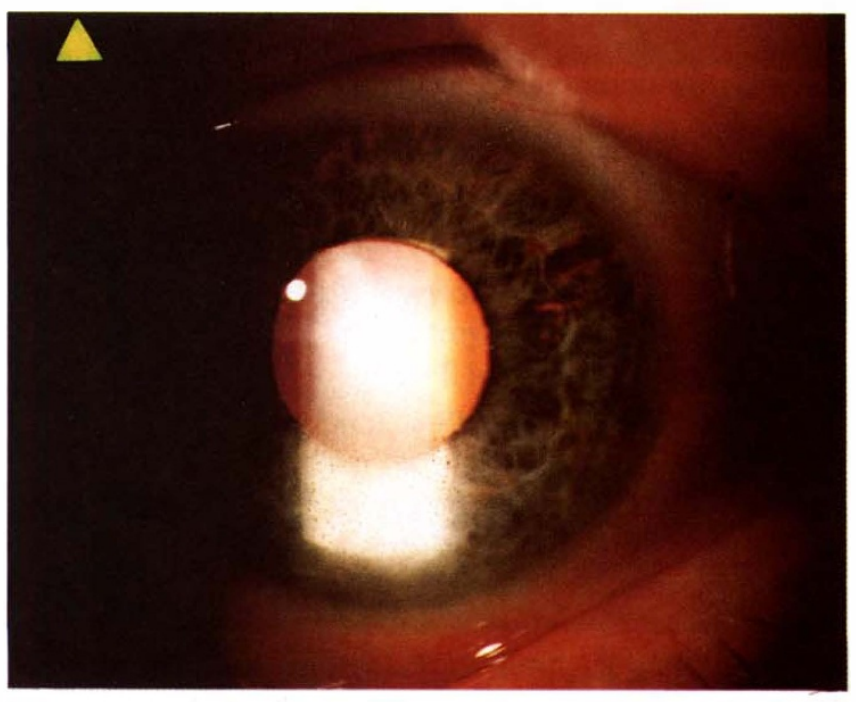

b

Fig. 3. Iris pigment epithelitis (case 1). (a) Pigmented keratic precipitates at presentation. (b) Mid-iridial pigment epithelial loss on transillumination. 

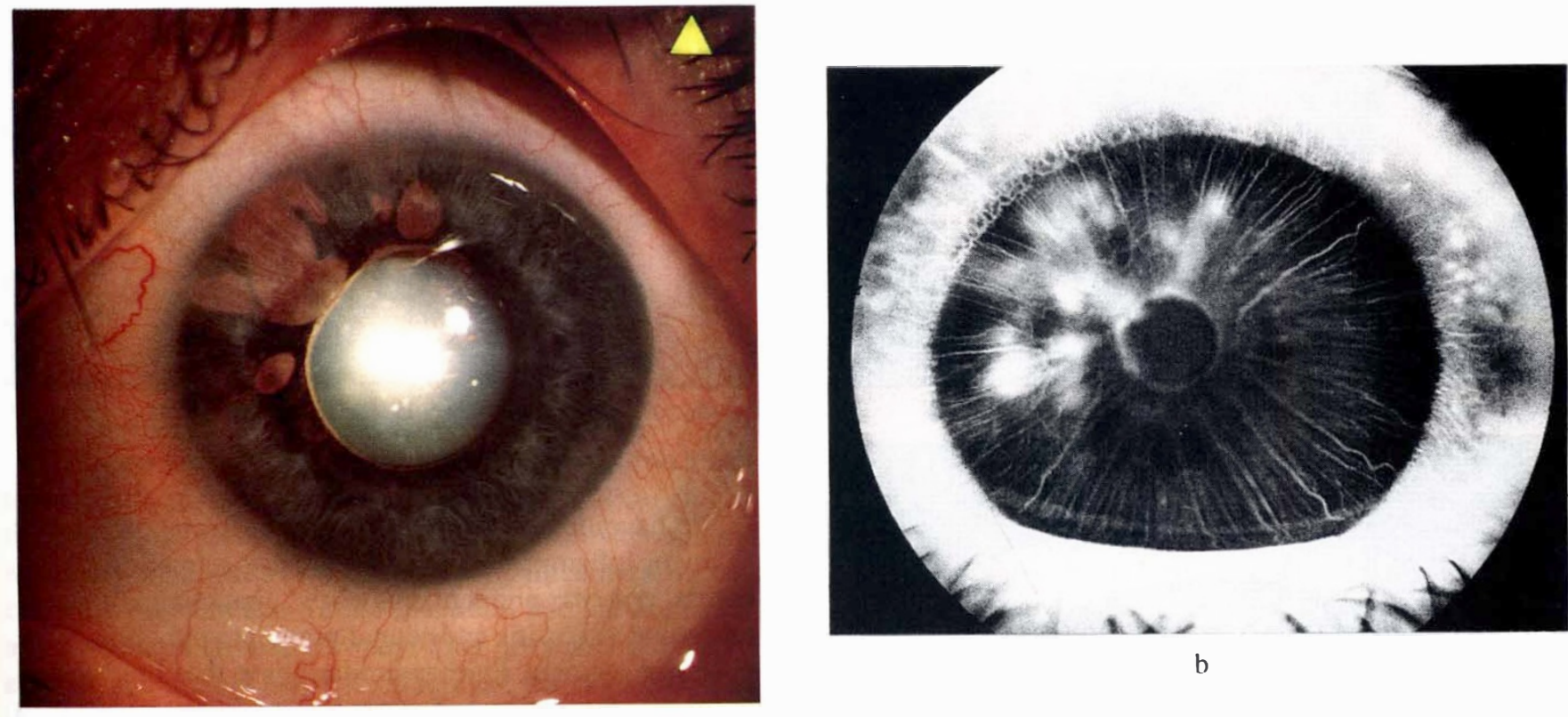

a

Fig. 4. Iris pigment epithelitis (case 2). (a) Confluent pigment epithelial loss on presentation of iritis. (b) Angiographic documentation of vascular leakage in the same quadrant as the iris pigment epithelial loss.

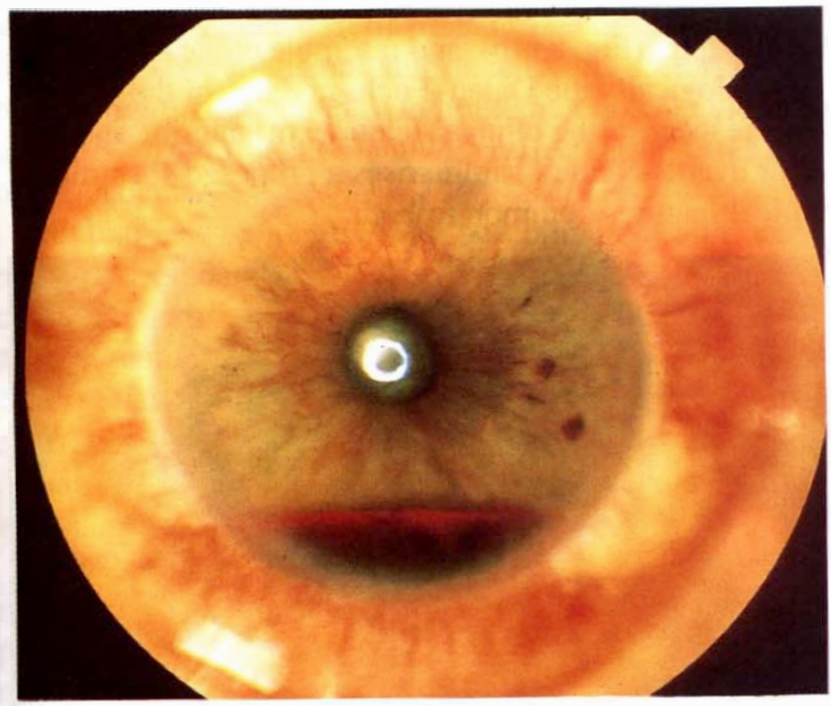

a

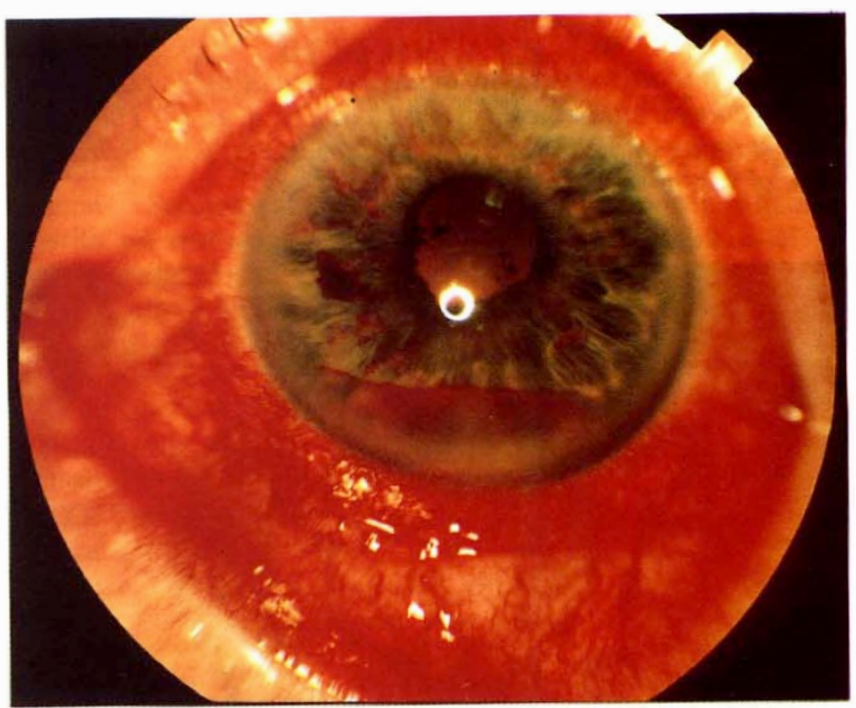

b

Fig. 5. Iris vasculitis. In both clinical photographs ( $a$ and $b$ ) there are coiled engorged vessels and associated hyphaemas. 


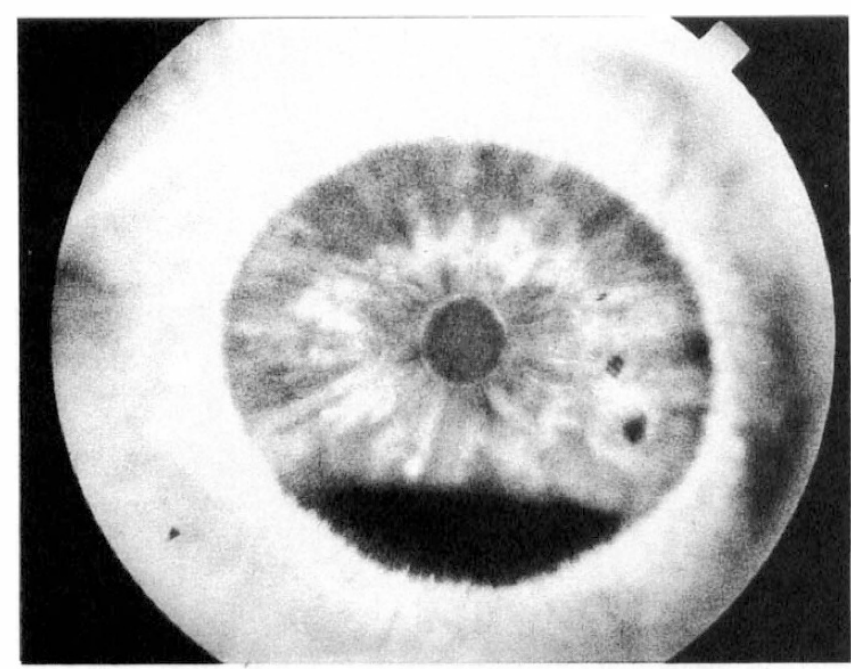

a

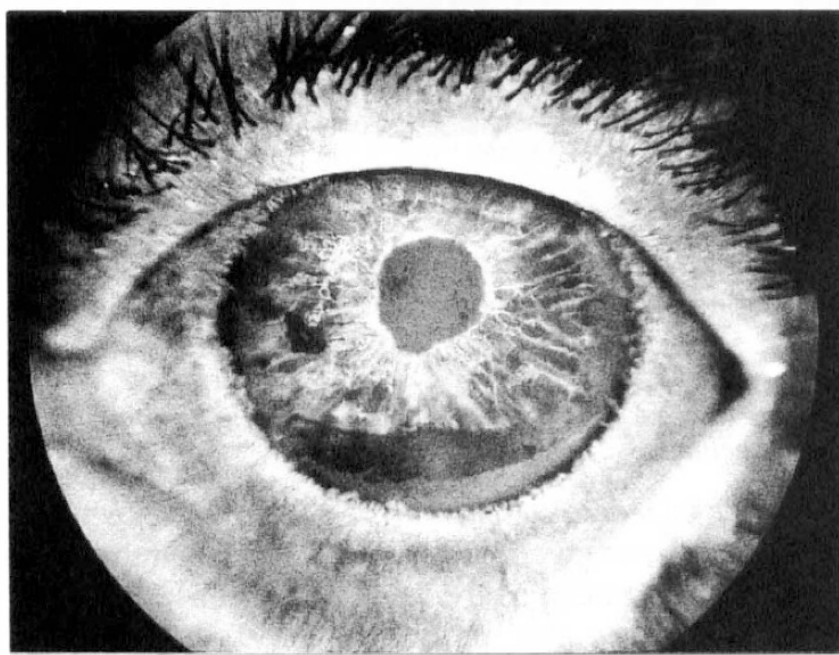

b

Fig. 6. Iris angiography of acute iris vasculitis. (a) Late leakage of fluorescein in the case shown in Fig. 5(a). (b) The vessel wall staining and early leakage of fluorescein, both characteristic of vasculitic vessels.

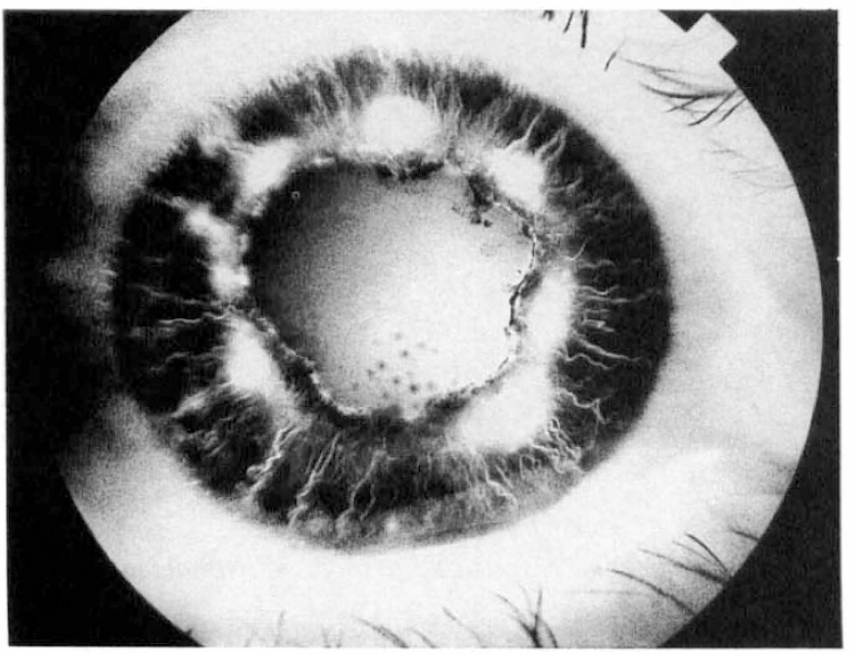

a

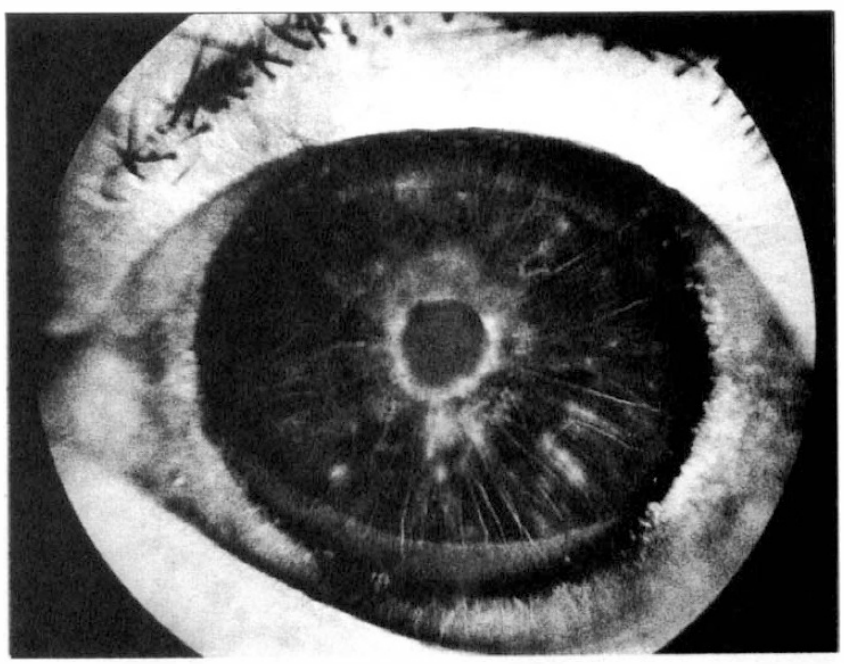

Fig. 7. Post-treatment iris angiography in iris vasculitis. The figure demonstrates the persistence of the leaking vessels.

We have demonstrated two clinical forms of anterior segment inflammation, which we have termed 'iris vasculitis' and 'iris pigment epithelitis', that clinically parallel retinal vasculitis and retinal pigment epithelitis in the posterior segment. Further similarities in the clinical presentation of anterior and posterior uveitis are also well known, such as the focal chorioretinal infiltrates of posterior uveitis (as occur in sympathetic ophthalmia and sarcoidosis) and the well-documented nodular leucocytic infiltrates in certain forms of anterior uveitis.

Granulomatous uveitis is a recognised type of iris inflammation in which leucocytic infiltrates can occur within the iris stroma (Koeppe and Busacca nodules) and microgranulomas are also found on the iris in sarcoid uveitis. ${ }^{9}$ Both of these features can be demonstrated by iris angiography (Fig. 8a, b), and resemble clinically and histopathologically the Dalen-Fuchs nodules of the choroid. With respect to the clinical features of anterior and posterior uveitis, Table I summarises a proposed spectrum of clinical features common to both.

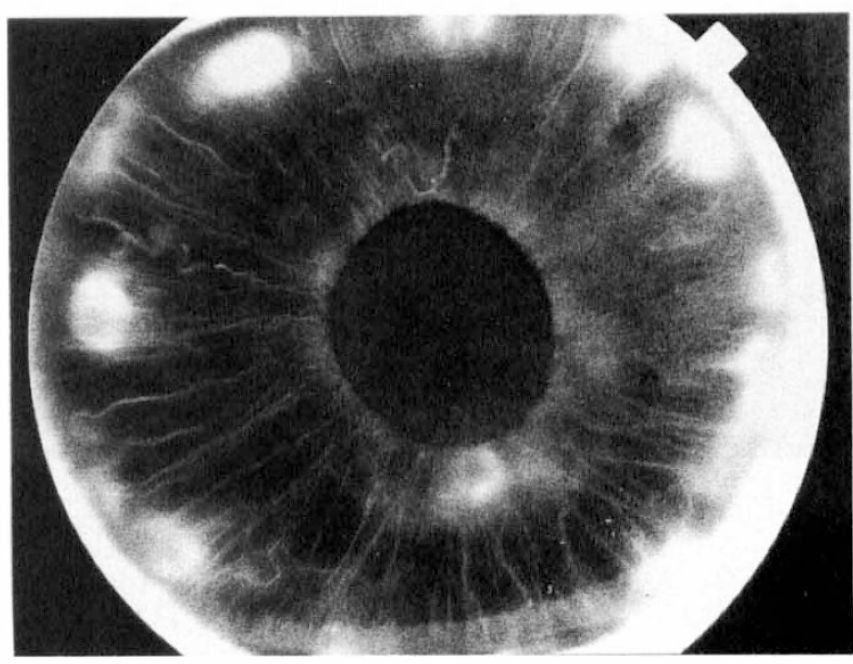

b

Fig. 8. Iris angiography in anterior uveitis. (a) Angiographic appearance of Busacca nodules. (b) Angiographic appearance of sarcoid microgranulomas. 
Table 1. Summary of the spectrum of clinical features adopted by anterior and posterior uveitis

\begin{tabular}{|c|c|c|c|c|}
\hline \multicolumn{2}{|c|}{ Posterior uveitis } & \multirow[b]{2}{*}{ Clinical signs } & \multicolumn{2}{|c|}{ Anterior uveitis } \\
\hline Types & & & & Types \\
\hline $\begin{array}{l}\text { Pars planitis } \\
\text { Intermediate uveitis }\end{array}$ & Vitritis & CELLS & $\begin{array}{l}\text { Anterior chamber } \\
\text { Keratic precipitates }\end{array}$ & $\begin{array}{l}\text { HLA B27+ } \\
\text { HLA B27- }\end{array}$ \\
\hline $\begin{array}{l}\text { Sympathetic ophthalmia } \\
\text { Sarcoidosis } \\
\text { Birdshot } \\
\text { APMPPE }\end{array}$ & Dalen-Fuchs nodules & FCRL & $\begin{array}{l}\text { Busacca nodules } \\
\text { Koeppe nodules } \\
\text { Pigment epithelitis }\end{array}$ & Sarcoid \\
\hline $\begin{array}{l}\text { Retinal vasculitis } \\
\text { peripheral } \\
\text { central } \\
\text { Behcet's }\end{array}$ & & VASCULITIS & Iris vasculitis & \\
\hline $\begin{array}{l}\text { VKH } \\
\text { Acute retinal necrosis }\end{array}$ & $\begin{array}{l}\text { Macular oedema } \\
\text { Retinal detachment }\end{array}$ & OEDEMA & $\begin{array}{l}\text { Cyclitis } \\
\text { Uveal effusion }\end{array}$ & \\
\hline
\end{tabular}

APMPPE, acute posterior multifocal placoid pigment epitheliopathy; FCRL, focal chorioretinal lesions; VKH, Vogt-Koyanaga-Harada syndrome.

The acute loss of iris pigment epithelium which we have termed 'iris pigment epithelitis' appears to occur in an otherwise low-grade inflammatory response. Although in 8 patients there was a history of herpetic infections, in our cases the pigment loss varied in extent, and did not have any associated stromal atrophy. This does not, however, exclude subclinical herpetic infections, but does identify that the iris pigment epithelium is involved in subgroups of anterior uveitis. In 2 of the patients we have been able to document the progress of this pigment loss during active inflammation. There was no evidence of concomitant iris vasculitis clinically, but fluorescein angiography on 1 patient did demonstrate concomitant vascular leakage, suggestive of iris vessel inflammation. However, as is seen in the iris vasculitis group of patients, vasculitis alone does not cause iris pigment epithelial loss. A similar finding has recently been described in 3 cases of unilateral iritis, ocular hypertension and patchy or sectorial iris pigment epithelial cell loss without any clinical evidence of herpes zoster infection but with raised serum titres in 2 of the patients. ${ }^{10}$

Iris pigment epithelitis may be compared with acute multifocal placoid pigment epitheliopathy (AMPPE), a clinical diagnosis of retinal pigment epithelitis, with perhaps a common underlying viral aetiology. The presence of sectorial vasculitis (angiographically) in one of the cases may also contribute to the loss of iris pigment epithelial cells, and further study of this group of patients with angiography is required.

Posterior uveitides exhibit varying degrees of vessel inflammation in both extent and severity and these may be primary or secondary. In our second group of anterior segment syndromes, patients presented with acute iritis, the predominant feature of which was that of iris vessel engorgement and hyphaema. Within this group there were other features attributed to an acute iritis, but despite the haemorrhage there was no rise in intraocular pressure. Fluorescein angiography confirmed vessel wall staining and leakage of fluorescein, similar to the appearances with primary retinal vasculitis. Haemorrhage and secondary glaucoma have been described previously in uveitis asso- ciated with herpes zoster, ${ }^{11}$ but our group of patients had no history of herpetic infections and no secondary glaucoma was present. As yet it is not possible to say whether the vessel abnormality is a primary vasculitis or secondary to the engorgement of the vessels and leakage as a consequence of liberated inflammatory mediators.

Although the majority of anterior uveitides are HLAB27+, other forms of iritis that we have described, which may involve subclinical herpetic infections, adopt clinical features similar to those seen in posterior uveitis, as summarised in Table I. Histopathological studies of these less common features of iritis are required to help a full understanding of the underlying immunopathogenesis of these disorders.

Key words: Iris pigment epithelitis, Iris vasculitis, Iritis, Posterior uveitis, Uveitis.

\section{REFERENCES}

1. Feltkemp TEW. Ophthalmological significance of HLA associated uveitis. Eye 1990;4:839-44.

2. Wakefield D, Stahlberg TH, Toivanen A, Granfers K, Tennant C. Serological evidence of Yersinia infection in patients with anterior uveitis. Arch Ophthalmol 1990;108:219-21.

3. Priem HA, Oosterhuis JA. Birdshot chorioretinopathy. Am J Ophthalmol 1988;105:182-5.

4. Forrester JV. Endogenous posterior uveitis. Br J Ophthalmol 1990;74:620-3.

5. Forrester JV. Uveitis: pathogenesis. Lancet 1992;338: 1489-501.

6. Forrester JV, Liversidge J, Dua HS, Towler H, McMenamin PG. Comparison of clinical and experimental uveitis. Curr Eye Res 1990;9:75-84.

7. Marsh RJ, Easty DL, Jones BR. Iritis and iris atrophy in herpes zoster ophthalmicus. Am J Ophthalmol 1974;94: 1899-902.

8. Shah SM, Spalton DJ, Smith SE. Measurement of aqueous cells and flare in normal eyes. Br J Ophthalmol 1991;75: 348-53.

9. Forrester JV. Sarcoidosis and inflammatory eye disease. Br J Ophthalmol 1992;76:193-4.

10. Couto C, Wainsztein R, Niveiro R, Alfonso G. Uveitis associated with ocular hypertension and iris atrophy: herpes zoster (abstract). In: Third international symposium on uveitis. 1992:174.

11. Karbassi M, Ralzman MB, Schuman JS. Herpes zoster ophthalmicus. Surv Ophthalmol 1992;36:395-410. 